\title{
Fontes orgânicas e inorgânicas de selênio na nutrição de vacas leiteiras: digestão, absorção, metabolismo e exigências
}

\author{
Organic and inorganic sources of selenium in the nutrition of dairy cows: digestion, absorption, \\ metabolism and requirements
}

\author{
Martin Gierus ${ }^{\mathrm{I}}$
}

\section{- REVISÃO BIBLIOGRÁFICA -}

\section{RESUMO}

A suplementação de bovinos leiteiros com selênio é feita tanto em sua forma orgânica como inorgânica. Nas plantas, o Se é incorporado aleatoriamente na sua forma orgânica e encontra-se como análogo de aminoácidos sulfurados, ou seja, selenometionina e selenocisteína. As formas inorgânicas, por sua vez, correspondem aos sais selenato $\left(\mathrm{SeO}_{4}^{-2}\right)$ e selenito $\left(\mathrm{SeO}_{3}{ }^{-2}\right)$. Contudo, enquanto a forma orgânica é metabolizada inicialmente como o aminoácido sulfurado análogo, as formas inorgânicas são metabolizadas diretamente via seleneto (HSe-) para serem incorporadas em selenoproteínas funcionais, como a glutationa peroxidase. Assim, a selenometionina é metabolizada como a metionina, sendo incorporada ao acaso em proteínas do organismo. Somente após o turnover protéico, a selenometionina é então liberada, podendo servir como fonte de Se. Além disso, em animais de alta produção, a incorporação de selenometionina a proteínas do leite pode torná-la indisponível às selenoproteínas funcionais. Em função disso, análises da concentração de Se no solo, nos alimentos dos animais e no leite, são somente complementares à análise da atividade da glutationa peroxidase, pois não indicam o grau com que o Se vai ser metabolizado pelo animal. O Se no leite, por sua vez, reflete a concentração de fontes orgânicas de Se no sangue. Em torno de $70 \%$ do Se no leite está incorporado à caseína. A suplementação de Se para bovinos leiteiros é necessária em qualquer fase de crescimento ou estado fisiológico dos animais, mas o metabolismo das diferentes fontes de Se precisa ser considerado. Enquanto que, em deficiências agudas, fontes inorgânicas parecem ser mais apropriadas, as fontes orgânicas podem ser eficientes em casos de deficiência crônica ou em períodos de suplementação ineficiente de Se.

Palavras-chave: ruminantes, selenometionina, selenoproteínas funcionais, glutationa peroxidase.

\section{ABSTRACT}

Selenium is supplemented for dairy cattle using organic or inorganic selenium sources. In plants, Se is randomly incorporated also in proteins and its organic form is found as an analogue of sulphur amino acids, i.e. selenomethionine (SeMet) or selenocystein (SeCis). Inorganic forms are mostly found as selenite $\left(\mathrm{SeO}_{3}^{-2}\right)$ or selenate $\left(\mathrm{SeO}_{4}^{-2}\right)$. However, it is important to distinguish between the metabolism of the different Se sources. Whereas the organic form (SeMet and SeCis) can be metabolized as amino acid, the inorganic forms are readily incorporated through selenide (HSe ${ }^{-}$into functional selenoproteins, like glutathione peroxidase. Thus, selenoamino acids are incorporated randomly to any body protein and are available as potential Se sources only after protein turnover. In addition, there is a risk for high yielding dairy cows to loose Se for functional selenoproteins when selenoamino acids, especially SeMet, are incorporated into milk proteins, considering that about $70 \%$ of Se in milk is incorporated to casein. Measurements of Se content in soil and feed samples have therefore only a complementary value, because the metabolism in dairy cows is difficult to be foreseen with only the Se content in soil and feeds. Se supplementation is important for all animals, but metabolism of the different Se sources needs to be taken into consideration. Whereas acute deficiencies are corrected with inorganic Se sources, organic sources seem to be more efficient to overcome temporarily deficiencies.

Key words: ruminants, selenomethionine, functional selenoproteins, glutathione peroxidase.

\section{INTRODUÇÃO}

O selênio (Se) é um microelemento essencial na dieta de animais e está envolvido no sistema

'Grassland and Forage Science/Organic Agriculture - Institute of Crop Science and Plant Breeding, Christian Albrechts University, D-24098 Kiel, Germany. E-mail: mgierus@email.uni-kiel.de. 
antioxidante do organismo através da enzima glutationa peroxidase (MILLER et al., 1993; ROVER Jr. et al., 2001). A glutationa peroxidase é uma metaloenzima com peso molecular de aproximadamente 80kDa (OH et al., 1974; ZACHARA, 1992) e contém Se em sua estrutura (4g de Se/mol de proteína).

Focos de deficiência de Se têm sido identificados em várias regiões do mundo, assim como no Brasil (MORAES et al., 1999), tornando indispensável a suplementação aos animais. Entretanto, a fonte de Se a ser suplementada apresenta diferenças se esta for orgânica ou inorgânica. Essas diferenças estão relacionadas ao diferente metabolismo inicial das duas formas do elemento.

Situações de deficiência de Se têm sido identificadas a campo pela medida de concentração do elemento no solo, nos alimentos, no sangue e no leite dos animais (LUCCI et al., 1984a; LUCCI et al., 1984b; WEISS et al., 1990; MALBE et al., 1995). Apesar de ser indicativa, a medida do Se total não permite que se faça diferença entre selenoproteínas funcionais e nãofuncionais presentes em uma amostra de alimento, sangue ou leite. Com isso, concentrações de Se no solo e/ou no alimento só podem ser vistas como medidas complementares.

O Se é um elemento que tem uma margem muito estreita entre os níveis de exigência e toxidez. Teores na dieta menores que $0,1 \mathrm{mg} \mathrm{kg}^{-1} \mathrm{MS}$ são considerados deficientes, e acima de $2 \mathrm{mg} \mathrm{kg}^{-1} \mathrm{MS}$ podem ser tóxicos. Contudo, em ruminantes, a atividade microbiana do rúmen influencia a forma com que o Se chega ao intestino delgado para absorção, diminuindo o risco de intoxicação (FISHER et al., 1980; MAUS et al., 1980; CRISTALDI et al., 2005). Além disso, o Se na forma orgânica existe como análogo de aminoácidos sulfurados, principalmente selenometionina (SeMet) e selenocisteína (SeCis). Como o Se está na mesma coluna da tabela periódica que o enxofre, os dois elementos têm muitas características em comum. Isso torna o Se um elemento químico único em seu metabolismo, se comparado a outros elementos como o zinco, cobre e cobalto (BURK et al., 2003). Assim, como análogo dos aminoácidos sulfurados, a forma orgânica de Se está presente como um produto direto da incorporação de Se em proteínas em substituição ao enxofre, o que difere das metaloproteínas ou quelatos, nos quais ocorre simplesmente uma complexação com grupos funcionais das proteínas (SUZUKI, 2005).

Sendo assim, a presente revisão bibliográfica tem por objetivo (1) caracterizar as formas de Se nos alimentos, (2) descrever o metabolismo ruminal e os mecanismos de absorção das diferentes formas de Se (orgânica ou inorgânica) em bovinos leiteiros, o metabolismo intermediário, o desempenho dos animais e (3) avaliar o uso das concentrações do Se no leite e sangue (ou plasma), assim como a atividade da enzima glutationa peroxidase, como indicadores da disponibilidade do elemento.

Selênio nos alimentos

A concentração de Se nas plantas forrageiras e grãos (concentrado) é dependente da sua concentração no solo, a qual pode variar pontualmente, ou seja, dentro de uma propriedade podem existir áreas com níveis normais de Se no solo, seguido de áreas adjacentes com deficiência do elemento. Isso pode afetar a ingestão total de Se pelos animais em pastejo (STEVENS et al., 1985). Em geral, pode-se afirmar que plantas crescendo em solos com baixo $\mathrm{pH}$, com altos teores de argila ou adubadas com produtos à base de sulfato, têm baixos teores de Se (SHAMBERGER, 1983; FOSTER \& SUMAR, 1995; KABATA-PENDIAS, 1998). Em contrapartida, plantas com maior massa radicular e raízes mais profundas, assim como plantas de crescimento mais lento e de maior teor protéico, tendem a possuir conteúdo mais alto de Se (SHAMBERGER, 1983; HARTFIEL \& SCHULTE, 1988). O farelo de soja pode apresentar teores de Se que variam de 0,20 e 0,40 mg Se $\mathrm{kg}^{-1} \mathrm{MS}$, enquanto que o milho moído ou o farelo de arroz podem conter níveis que variam de 0,01 a $0,05 \mathrm{mg}$ Se $\mathrm{kg}^{-1} \mathrm{MS}$. A silagem de milho, por sua vez, apresenta teores de Se menores, em comparação a gramíneas forrageiras (SCHÄFER \& WOLLGIEN, 1986; GIERUS et al., 2002). O teor de Se em pastagens também é bastante variável. Enquanto que GRACE et al. (1997) verificaram em pastagens na Nova Zelândia teores de

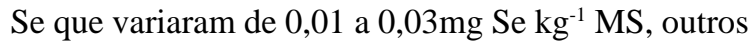
autores determinaram concentrações de Se em amostras de pastagens temperadas que variaram de 0,03 a $0,12 \mathrm{mg}$ Se kg-1 MS (MEYER et al., 1995; GIERUS et al., 2002).

Embora tenha-se observado grande variação no teor de Se em volumosos e concentrados, a concentração em plantas e grãos é baixa. A ingestão de Se através do conteúdo natural de Se nas plantas e, posteriormente, como componentes em dietas (Tabela 1) é insuficiente para cobrir a exigência nutricional do elemento para bovinos leiteiros em quaisquer das fases de crescimento. Uma suplementação com misturas minerais contendo Se é, portanto, indispensável.

Nas plantas, o Se parece não exercer nenhuma função fisiológica (TERRY et al., 2000), e é incorporado aleatoriamente no tecido vegetal em ambas as formas: orgânica e inorgânica. As principais formas de Se inorgânico são os sais de Se, ou seja, selenato $\left(\mathrm{SeO}_{4}^{-2}\right)$ e selenito $\left(\mathrm{SeO}_{3}^{-2}\right)$. Por sua vez, a forma orgânica mais comum do Se na planta é a SeMet ou SeCis, ou 
Tabela 1 - Efeitos da suplementação de selênio sobre o desempenho animal e a concentração de selênio no leite, sangue ou plasma.

\begin{tabular}{|c|c|c|c|c|c|c|}
\hline Autor & $\begin{array}{l}\text { Teor de Se na ração* } \\
\quad\left(\mathrm{mg} \mathrm{kg}^{-1} \mathrm{MS}\right)\end{array}$ & $\begin{array}{l}\text { Suplementação de Se } \\
\text { (forma química) }\end{array}$ & $\begin{array}{l}\text { Duração do } \\
\text { experimento }\end{array}$ & $\begin{array}{l}\text { Desempenho } \\
\left(\mathrm{kg} \text { leite } \mathrm{dia}^{-1}\right)\end{array}$ & & $\begin{array}{l}\text { r de Se } \\
\text { Se } l^{-1} \text { ) }\end{array}$ \\
\hline \multirow{6}{*}{ 1) } & \multirow{6}{*}{0,200} & & \multirow{6}{*}{13 semanas } & \multirow{6}{*}{ s.i. } & leite & $\begin{array}{l}\text { Sangue/plasma } \\
\text { (plasma) }\end{array}$ \\
\hline & & $\left(\mathrm{Na}_{2} \mathrm{SeO}_{3}\right)$ & & & & \\
\hline & & $0 \mathrm{mg} \mathrm{dia}{ }^{-1}$ & & & 49 & 117 \\
\hline & & $2 m g$ dia $^{-1}$ & & & 56 & 111 \\
\hline & & $4 \mathrm{mg} \mathrm{dia}{ }^{-1}$ & & & 56 & 120 \\
\hline & & $10 \mathrm{mg} \mathrm{dia}^{-1}$ & & & 55 & 126 \\
\hline \multirow{4}{*}{ 2) } & \multirow{4}{*}{0,180} & $\left(\mathrm{Na}_{2} \mathrm{SeO}_{3}\right)$ & \multirow{4}{*}{5 dias } & & & (plasma) \\
\hline & & $0 \mathrm{mg} \operatorname{dia}^{-1}$ & & 21,5 & 28 & 132 \\
\hline & & $6 m g$ dia $^{-1}$ & & 23,8 & 28 & 128 \\
\hline & & $100 \mathrm{mg} \mathrm{dia}^{-1}$ & & 24,8 & 33 & 147 \\
\hline \multirow{5}{*}{ 3) } & \multirow{5}{*}{ s.i. } & $\left(\mathrm{Na}_{2} \mathrm{SeO}_{3}\right)$ & \multirow{5}{*}{8 semanas } & \multirow{5}{*}{ s.i. } & & (sangue) \\
\hline & & $0 \mathrm{mg} \mathrm{kg}^{-1} \mathrm{MS}$ & & & 3,2 & 6,6 \\
\hline & & $\begin{array}{l}0,2 \mathrm{mg} \mathrm{kg}^{-1} \mathrm{MS} \\
\text { (LE) }\end{array}$ & & & 23,7 & 90,7 \\
\hline & & $0 \mathrm{mg} \mathrm{kg}^{-1} \mathrm{MS}$ & & & 3,2 & 5,1 \\
\hline & & $0,2 \mathrm{mg} \mathrm{kg}^{-1} \mathrm{MS}$ & & & 63,6 & 167,3 \\
\hline \multirow{3}{*}{ 4) } & \multirow{3}{*}{0,110} & $\left(\mathrm{Na}_{2} \mathrm{SeO}_{3}\right)$ & \multirow{3}{*}{32 dias } & & & (plasma) \\
\hline & & $2,0 \mathrm{mg} \mathrm{dia}{ }^{-1}$ & & 33,8 & s.i. & 55,0 \\
\hline & & $6,6 \mathrm{mg} \mathrm{dia}{ }^{-1}$ & & 32,8 & & 115,0 \\
\hline \multirow{4}{*}{ 5) } & \multirow{4}{*}{0,035} & $\left(\mathrm{Na}_{2} \mathrm{SeO}_{4}\right)$ & \multirow{4}{*}{19 semanas } & \multirow{4}{*}{ s.i. } & & (sangue) \\
\hline & & 0mg dia ${ }^{-1}$ & & & 8 & 20 \\
\hline & & $2 \mathrm{mg} \mathrm{dia}^{-1}$ & & & 14 & 95 \\
\hline & & $4 \mathrm{mg} \operatorname{dia}^{-1}$ & & & 16 & 157 \\
\hline \multirow{4}{*}{ 6) } & \multirow{4}{*}{0,038} & $\left(\mathrm{Na}_{2} \mathrm{SeO}_{3}\right)$ & \multirow{4}{*}{6 semanas } & & & (plasma) \\
\hline & & $0 \mathrm{mg} \mathrm{dia}{ }^{-1}$ & & 20,1 & 9,9 & 19 \\
\hline & & $1,6 \mathrm{mg} \mathrm{dia}{ }^{-1}$ & & 20,7 & 9,5 & 47 \\
\hline & & $5,6 \mathrm{mg} \mathrm{dia}^{-1}$ & & 20,2 & 14,7 & 56 \\
\hline \multirow{4}{*}{ 6) } & \multirow{4}{*}{0,043} & $\left(\mathrm{Na}_{2} \mathrm{SeO}_{3}\right)$ & \multirow{4}{*}{6 semanas } & & & (plasma) \\
\hline & & $0 \mathrm{mg} \mathrm{dia}^{-1}$ & & 16,6 & 6,5 & 13 \\
\hline & & $1,6 \mathrm{mg} \mathrm{dia} a^{-1}$ & & 16,5 & 7,3 & 16 \\
\hline & & $5,6 \mathrm{mg} \mathrm{dia}^{-1}$ & & 16,9 & 10,6 & 58 \\
\hline \multirow{4}{*}{ 6) } & \multirow{4}{*}{0,054} & $\left(\mathrm{Na}_{2} \mathrm{SeO}_{3}\right)$ & \multirow{4}{*}{6 semanas } & & & (plasma) \\
\hline & & $0 \mathrm{mg} \operatorname{dia}^{-1}$ & & 27,3 & 15,3 & 32 \\
\hline & & $1,6 \mathrm{mg} \mathrm{\textrm {dia } ^ { - 1 }}$ & & 27,4 & 15,8 & 50 \\
\hline & & $5,6 \mathrm{mg} \mathrm{dia}{ }^{-1}$ & & 27,8 & 20,1 & 71 \\
\hline
\end{tabular}

s.i.: sem indicação; LE=levedura enriquecida, rico em formas orgânicas de selênio.

* concentração de selênio na ração basal.

Autores: 1) MAUS et al. (1980); 2) FISHER et al. (1980); 3) MALBE et al. (1995); 4) WEISS et al. (1990); 5) KNOWLES et al. (1999); 6) GIERUS et al. (2002).

seja, uma forma análoga dos aminoácidos sulfurados. Na estrutura molecular dos aminoácidos, o Se ocupa o mesmo lugar que o enxofre (Figura 1). Algumas plantas apresentam mais de 50\% do Se na forma orgânica. Em milho, trigo e soja crescendo em solos ricos em Se, a SeMet pode representar mais de $80 \%$ do Se total presente no tecido vegetal (YANG et al., 1997; GUO \& WU, 1998).

Ciência Rural, v.37, n.4, jul-ago, 2007. 


\begin{tabular}{|l|}
\hline \\
Figura 1 - Estrutura molecular da metionina e selenometionina, \\
mostrando a localização dos átomos de selênio e \\
enxofre. A substituição do átomo de selênio por enxofre \\
na estrutura molecular da selenocisteína ocorre de modo \\
similar ao observado entre metionina e \\
selenometionina.
\end{tabular}

Os suplementos minerais também possuem Se tanto na forma inorgânica como orgânica. A forma inorgânica mais comum utilizada em misturas minerais é o selenito de sódio. As formas orgânicas, por sua vez, estão disponíveis como leveduras enriquecidas, que crescem sobre um substrato contendo pouco enxofre e muito Se. Dessa forma, o Se encontrado é basicamente a SeMet, porém com diversas outras formas ainda desconhecidas (UDEN et al., 2004).

Fermentação ruminal e absorção de selênio

Selenito e selenato podem ser reduzidos a Se elementar $\left(\mathrm{Se}^{0}\right)$ pelos microrganismos ruminais como um meio de detoxificação (KIM et al., 1997). Os microrganismos podem também incorporar Se na proteína microbiana(HIDIROGLOU \& LESSARD, 1976; KIM et al., 1997). Desse modo, o Se na ração, presente na forma inorgânica, pode ser absorvido no intestino delgado na forma orgânica. A composição da dieta pode influenciar a forma de Se que chega ao intestino delgado. A relação volumoso/concentrado, por exemplo, parece ter influência por afetar a composição da população microbiana ruminal (KOENIG et al., 1997; GIERUS et al., 2002). Contudo, tem sido pouco documentado, como as formas de Se que chegam ao intestino delgado são afetadas pela forma e pela quantidade de Se suplementada, assim como pelo ambiente ruminal.

A absorção intestinal de Se em ruminantes é semelhante à de monogástricos. A absorção de selenito é por difusão e, portanto, proporcional à quantidade presente no lúmen intestinal. A absorção de selenato e SeMet ocorre por transporte ativo, com o selenato sendo absorvido de modo semelhante ao sulfato e a SeMet de forma análoga à metionina (VENDELAND et al., 1994). Pouco do Se elementar é absorvido no intestino devido a sua baixa solubilidade e, portanto, passa a ser excretado nessa forma pelas fezes.

Metabolismo, exigências e excreção de selênio Após a absorção intestinal, SeMet e SeCis podem ser metabolizadas pelos animais como aminoácidos (EKHOLM et al., 1991). Isso ocorre especialmente com a SeMet, que, de forma similar à metionina, pode ser incorporada a proteínas pelo mesmo códon AUG, pois o tRNA não diferencia a metionina da SeMet. Em consequência, 40-50\% do Se corporal podem ser SeMet inserida em proteínas do tecido muscular, denominadas selenoproteínas não-funcionais (DANIELS, 1996).

No metabolismo pós-absortivo das formas inorgânicas, o selenito é captado pelos eritrócitos, reduzido imediatamente a seleneto $\left(\mathrm{HSe}^{-}\right)$, acoplado à albumina e transferido ao plasma, para então ser transportado ao fígado. O selenato, por sua vez, é incorporado diretamente pelos hepatócitos, utilizando o mesmo sistema de transporte dos fosfatos da corrente sanguínea (DANIELS, 1996; SUZUKI, 2005). Seleneto também resulta diretamente da atividade da ß-liase, enquanto que a SeMet é transformada a SeCis através de uma rota semelhante à transsulfuração, que ocorre entre metionina e cisteína (BUTLER \& WHANGER, 1989).

O metabolismo pós-absortivo de Se envolve dois compartimentos (Figura 2, JANGHORBANI et al., 1990; SUZUKI, 2005). Um compartimento contém as formas orgânicas de Se, representado principalmente pela SeMet e pela SeCis; e o outro, as formas inorgânicas. Para que o Se seja incorporado especificamente em selenoproteínas funcionais, como a glutationa persoxidase, é necessário que as formas orgânicas e inorgânicas sejam reduzidas a seleneto, e este, por sua vez, metabolizado a SeCis. Para tal, o seleneto reage com ATP, formando selenofosfato, numa reação catalisada pela selenofosfato sintetase. A seguir, junto com um resíduo de serina, o selenofosfato forma uma SeCis, que é pós-translacionalmente inserida nas selenoproteínas funcionais através de um códon UGA específico (SUNDE \& HOEKSTRA, 1980; DRISCOLL \& COPELAND, 2003). A SeCis da dieta, por sua vez, não é incorporada diretamente em selenoproteínas funcionais, necessitando ser inicialmente reduzida a seleneto. As formas inorgânicas são mais rapidamente 


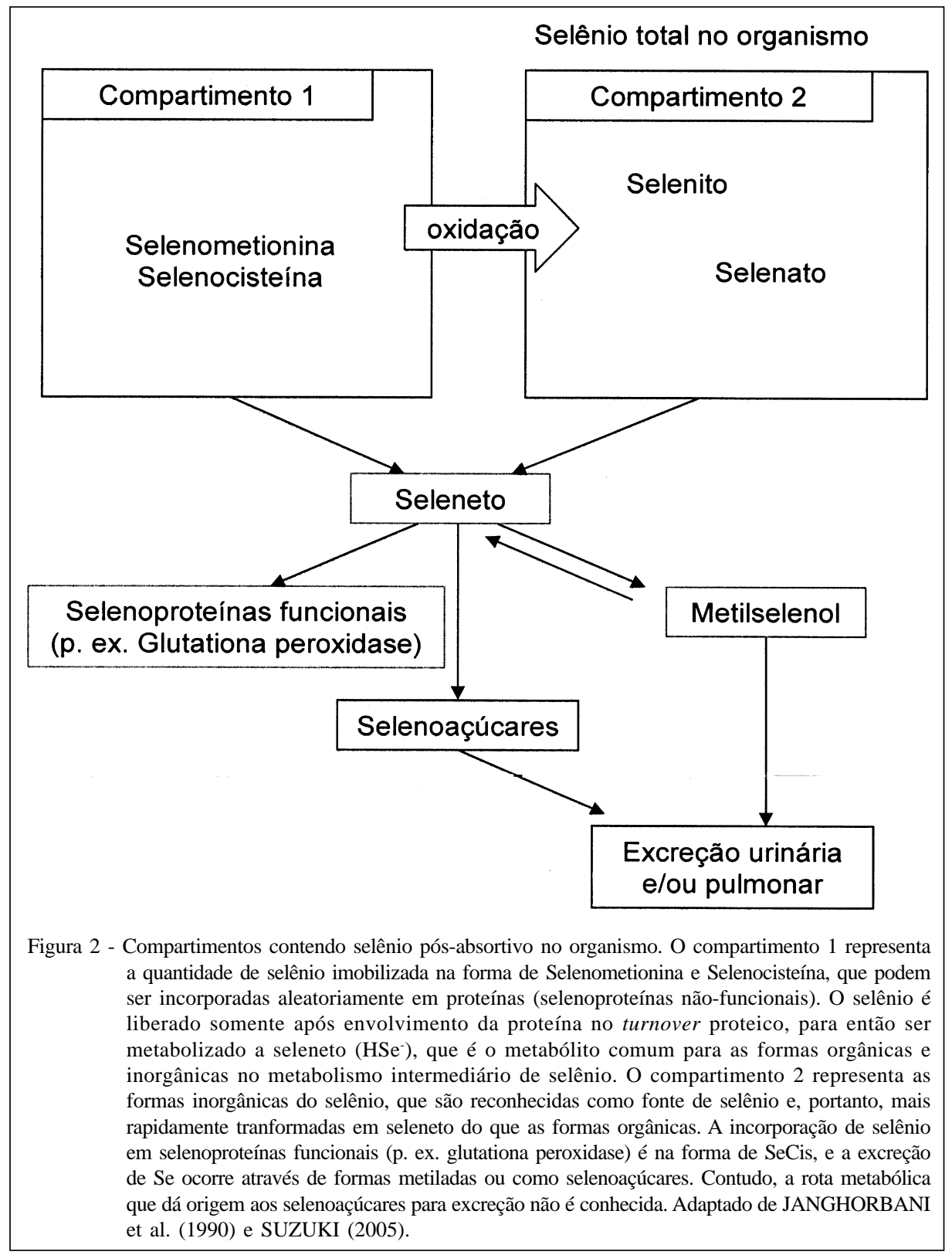

transformadas em seleneto que as orgânicas. SeMet e SeCis podem ser oxidadas a selenito ou selenato. A SeMet só é utilizada como fonte de Se pelo organismo após a degradação das proteínas em que ela foi incorporada, desde que estas proteínas sejam recicladas no interior das células. Formas orgânicas de Se incorporadas em proteínas não-funcionais, como em proteínas da lã, pêlos, cascos ou leite, são irreversivelmente perdidas. A SeMet pode ser transformada a SeCis pela mesma rota que a metionina é convertida a cisteína.

Atualmente, a maior parte das recomendações para suplementação de Se para bovinos leiteiros varia entre 0,1 a $0,3 \mathrm{mg} \mathrm{kg}^{-1} \mathrm{MS}$, independentemente do estado fisiológico, da idade dos animais ou da forma com que o Se é suplementado (ARC, 1980; GfE, 2001; NRC, 2001). O método clássico de determinação das exigências nutricionais implica a oferta de doses crescentes do elemento em questão. $\mathrm{O}$ parâmetro a ser medido como resposta deve reagir proporcionalmente e, atingido um ponto de equilíbrio, há uma estagnação na resposta do parâmetro medido (Figura 3). No caso do Se, a determinação da exigência difere do método clássico. A princípio, o metabolismo do Se parece não ser regulado homeostaticamente como o de outros microminerais. Uma das razões é a 


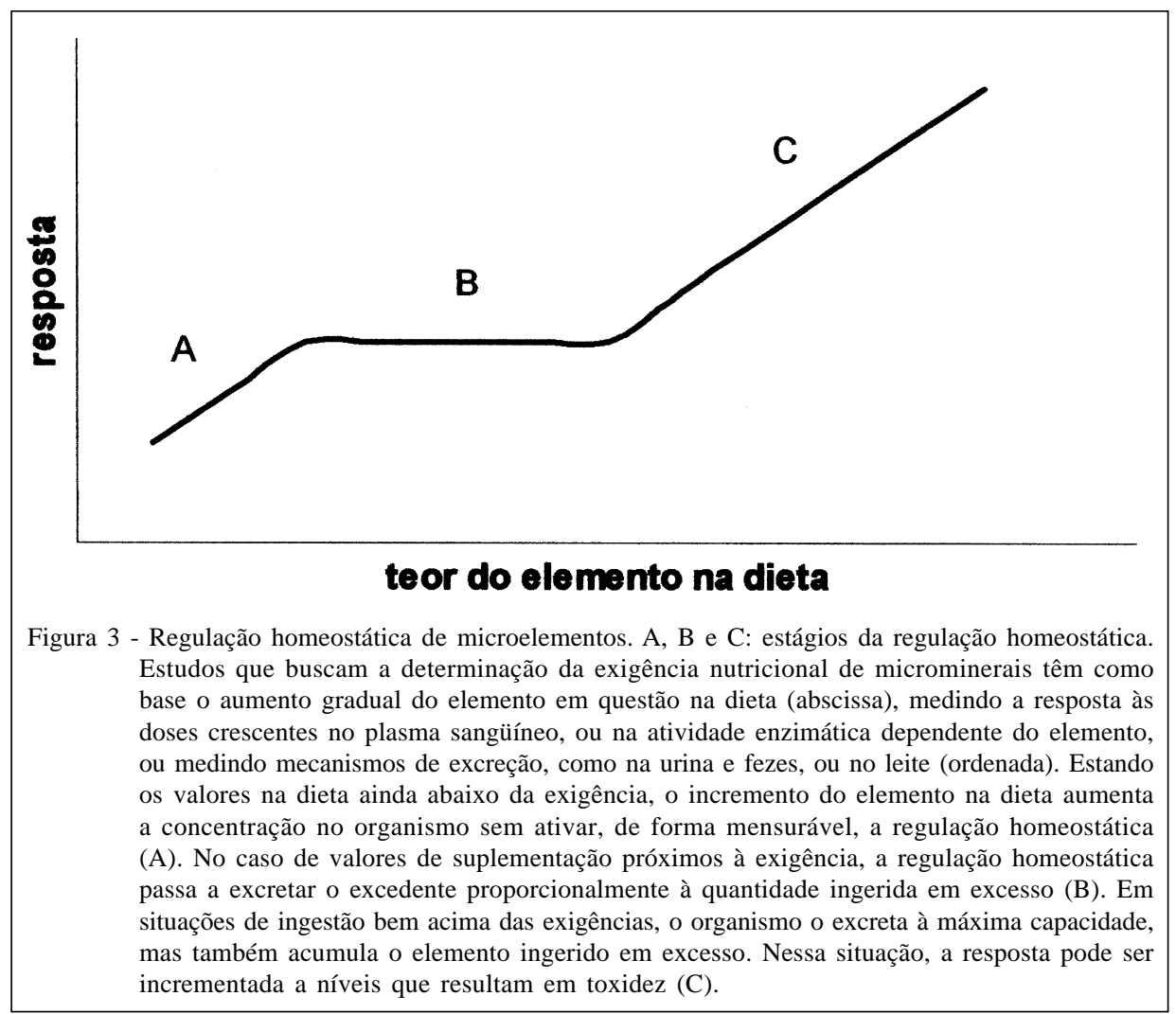

falta de dados na literatura que sugiram a existência de reservas de Se no organismo. No caso de deficiência, estas seriam ativadas, suprindo as exigências do elemento.

Como o metabolismo dos microminerais nos animais possui um controle homeostático, o organismo regula situações de deficiência, através da mobilização de reservas, e de excesso, através da excreção dos excedentes ou da redução da absorção intestinal (KIRCHGESSNER, 1993; KIRCHGESSNER et al., 1997; SCHWARZ et al., 2000). No entanto, diferente do que ocorre com outros microelementos, parece não existir regulação da absorção intestinal das diferentes formas de Se (VENDELAND et al., 1994). A excreção de excedentes de Se, por sua vez, ocorre pela transformação do elemento em formas metiladas menos tóxicas que selenito, selenato e selenoaminoácidos, como o trimetilselenônio ou selenoaçúcares (FRANCESCONI \& PANNIER, 2004), os quais são eliminados do organismo por via urinária. Em casos de níveis muito elevados de ingestão de Se, a via respiratória passa a ser uma rota complementar de excreção do elemento na forma metilada. Adicionalmente, a ingestão excessiva das formas orgânicas de Se pode resultar num aumento da sua incorporação nas proteínas do organismo, podendo, inclusive, alterar a função destas. Casos de toxidez são mais raros que casos de deficiência de Se em animais domésticos. Sintomas de tais situações são abordados em uma forma mais detalhada em PULS (1994) e UNDERWOOD \& SUTTLE (1999).

\section{Suplementação com selênio e resposta animal}

LACETERA et al. (1996) verificaram aumentos significativos na produção de leite, de 24,5 para $27,7 \mathrm{~kg} \mathrm{dia}^{-1}, 12$ semanas pós-parto, em vacas suplementadas com $5 \mathrm{mg} 100 \mathrm{~kg}^{-1}$ de peso vivo de selenito de sódio. Efeito positivo da suplementação com Se na produção de leite também foi verificado por FISHER et al. (1980). No entanto, a suplementação com Se nem sempre resulta em aumento da produção animal (WEISS et al., 1990; GIERUS et al., 2002). GIERUS et al. (2002) verificaram que a suplementação de vacas de leite com selenito de sódio influenciou o teor de Se no leite e plasma (Tabela 1), mas não houve incrementos na produção de leite, assim como não houve alteração na composição do leite. A atividade da glutationa peroxidase não aumentou significativamente além da dose de Se considerada adequada para bovinos leiteiros, representada pelo segundo nível de suplementação neste experimento, que foi de $0,20 \mathrm{mg}$ Se $\mathrm{kg}^{-1} \mathrm{MS}$ (GfE, 2001).

Ciência Rural, v.37, n.4, jul-ago, 2007. 
De outra forma, GIERUS et al. (2003) demonstraram que vacas suplementadas no período seco tinham quantidades de Se no plasma sangüíneo até 21 dias após o parto maiores que vacas nãosuplementadas, mesmo com a interrupção da suplementação após o parto. O teor de Se no colostro foi maior nas vacas suplementadas no período seco, mas não diferiu no leite 7 ou 21 dias após o parto, em comparação às não-suplementadas. A atividade da glutationa peroxidase também foi maior no período pósparto nas vacas suplementadas no período seco. Isso indica que, com o parto e a interrupção da suplementação, a atividade da enzima foi mantida com base em reservas de Se disponíveis, provavelmente pela liberação de Se das formas orgânicas através do turnover proteico. Contudo, a produção de leite, medida aos 21 dias pós-parto, não foi afetada pela suplementação com Se no período seco.

Experimentos de longa duração comparando suplementação com diferentes formas de Se durante um ou mais ciclos de lactação são raros (WEISS et al., 1990). A maioria dos estudos têm sido conduzidos em períodos experimentais relativamente curtos (Tabela 1). Nessas situações, o efeito residual da ingestão de Se em períodos anteriores pode ter interferido nos resultados desses estudos. Considerando que o Se pode ser incorporado aleatoriamente em proteínas corporais, qualquer resultado experimental de suplementação com Se tem que levar em consideração o período anterior ao seu início. O ideal é que, num período pré-experimental, os animais recebam dietas com forragens contendo baixos teores de Se. De qualquer maneira, esses estudos têm demonstrado uma maior eficiência de formas orgânicas do Se em aumentar a concentração de Se no sangue e leite de vacas, se comparado às inorgânicas (Tabela 1). Contudo, maior concentração destas formas nas dietas de vacas em lactação de alta produção também implica maiores perdas através da excreção de Se pela glândula mamária, através da caseína e de outras proteínas do leite, que incorporam aleatoriamente SeMet da dieta (MALBE et al., 1995; ORTMAN \& PEHRSON, 1997; KNOWLES et al., 1999; MUÑIZ-NAVEIRO et al., 2005). Nesta situação, a proporção que sobra para o animal suprir suas exigências por Se, ou seja, para manter ativas e em normal funcionamento as selenoproteínas funcionais, não é conhecida. O organismo de vacas de alta produção regula o metabolismo para maximizar o aporte de nutrientes para a secreção de leite (BAUMAN, 2000) e, em consequência, formas orgânicas de Se podem ser excretadas no leite em maior quantidade. Desse modo, a probabilidade de animais de alta produção apresentarem sintomas de deficiência de Se é mais alta.
Em algumas situações, pode ser vantajoso aumentar a quantidade total de Se através da suplementação de Se na forma orgânica (MALBE et al., 1995). No entanto, a incorporação indiscriminada de SeMet em selenoproteínas não-funcionais pode influenciar a função de proteínas que dependem da existência, por exemplo, de pontes de dissulfeto em sua estrutura.

Indicadores do status metabólico do selênio

A selenoproteína funcional mais comumentemente utilizada para estudar a resposta à suplementação de Se em animais é a glutationa peroxidase (ZACHARA, 1992; ARTHUR \& BECKET, 1994). Esta enzima está envolvida no sistema antioxidante do organismo, que neutraliza a produção excessiva de peróxidos e radicais livres, responsáveis pelo estresse oxidativo (MILLER et al., 1993; BRZEZINSKA-SLEBODZINSKA et al., 1994; NOCKELS, 1996). No período periparturiente, menor frequência das infecções intrauterinas e na glândula mamária têm sido relacionadas com a suplementação de Se e vitamina E. Na lactação, a maior incidência de alta contagem de células somáticas tem sido relacionada com a deficiência de Se (JUKOLA et al., 1996; PASCHOAL et al., 2003).

Outras formas de selenoproteínas funcionais são as deiodinases, envolvidas na atividade da tireóide, e também as selenoproteínas $\mathrm{W}$ e $\mathrm{P}$, encontradas em vários tecidos. As funções destas últimas, contudo, não estão claramente definidas (BEILSTEIN et al., 1996; BURK et al., 2003).

A medida do teores de Se em suas diferentes formas, na dieta, no sangue ou leite, fornece informações complementares, porém incompletas, do metabolismo do Se em vacas de leite. A medida da atividade de selenoproteínas funcionais parece ser o melhor indicador do status metabólico e o melhor critério para definir estratégias de suplementação com Se. No entanto, um método prático e padrão para esta análise ainda não foi proposto.

\section{CONCLUSÕES}

A ingestão e absorção de Se pelos ruminantes pode ser nas formas orgânica e inorgânica. As formas orgânicas, especialmente a SeMet, precisam ser liberadas de proteínas (selenoproteínas nãofuncionais) onde foram incorporadas para que o Se possa ser metabolizado via seleneto para selenoproteínas funcionais, como a glutationa peroxidase. Concentrações de Se no sangue refletem a concentração total do elemento no organismo, não 
fazendo, contudo, distinção entre formas orgânica e inorgânica. A concentração de Se no leite, por sua vez, reflete a quantidade de Se na forma orgânica que foi suplementada ou convertida pelos microrganismos ruminais. A concentração do Se no leite não indica a disponibilidade de Se para as selenoproteínas funcionais. A relação entre a concentração de Se no sangue ou plasma com a atividade da glutationa peroxidase somente é alta em animais deficientes em Se. A medida da atividade da gutationa peroxidase é o melhor indicador do status metabólico do Se em vacas de leite.

\section{REFERÊNCIAS}

ARC, Agricultural Research Council. The nutrient requirements of ruminant livestock. London, UK, 1980. 351p.

ARTHUR, J.R.; BECKETT, G.J. New metabolic roles for selenium. Proceedings of the Nutrition Society, v.53, p.615624, 1994.

BAUMAN, D.E. Regulation on nutrient partitioning during lactation: homeostasis and homeorhesis revisited. In: CRONJÉ, P.B. Ruminant physiology - digestion, metabolism, growth and reproduction. Wallingford: CABI, 2000. Cap.18, p.311-328.

BEILSTEIN, M.A. et al. Selenoprotein $\mathrm{W}$ of rat muscle binds glutathione and an unknown small molecular weight moiety. Journal of Inorganic Biochemistry, v.61, p.117-124, 1996

BRZEZINSKA-SLEBODZINSKA, E. et al. Antioxidant status of dairy cows supplemented prepartum with vitamin $\mathrm{E}$ and selenium. Journal of Dairy Science, v.77, p.3087-3095, 1994.

BURK, R.F. et al. Selenoprotein metabolism and function: evidence of more than one function for selenoprotein P. Journal of Nutrition, v.133, p.1517S-1520S, 2003.

BUTLER, J.A.; WHANGER, P.D. Influence of dietary methionine on the metabolism of selenomethionine in rat. Journal of Nutrition, v.119, p.1001-1009, 1989.

CRISTALDI, L.A. et al. Tolerance of inorganic selenium in wether sheep. Small Ruminant Research, v.56, p.205213, 2005.

DANIELS, L.A. Selenium metabolism and bioavailability. Biological Trace Element Research, v.54, p.185-199, 1996.

DRISCOLL, D.M.; COPELAND, P.R. Mechanism and regulation of selenoprotein synthesis. Annual Review of Nutrition, v.23, p.17-40, 2003.

EKHOLM, P. et al. Transport of feed selenium to different tissues of bulls. British Journal of Nutrition, v.66, p.49-55, 1991

FISHER, L.J. et al. The effect of added dietary selenium on the selenium content of milk, urine and feces. Canadian Journal of Animal Science, v.60, p.79-86, 1980.
FOSTER, L.H.; SUMAR, S. Methods of analysis used for the determination of selenium in milk and infant formulae: a review. Food Chemistry, v.53, p.453-466, 1995.

FRANCESCONI, K.A.; PANNIER, F. Selenium metabolites in urine: a critical overview of past work and current status. Clinical Chemistry, v.50, p.2240-2253, 2004.

GfE, Gesellschaft für Ernährungsphysiologie. Empfehlungen zur Energie- und Nährstoffversorgung der Milchkühe und Aufzuchtrinder. DLG Verlag: Frankfurt am Main, 2001. $136 p$.

GIERUS, M. et al. Selenium supplementation and selenium status of dairy cows fed diets based on grass, grass silage or maize silage. Journal of Animal Physiology and Animal Nutrition, v.86, p.74-82, 2002.

GIERUS, M. et al. Untersuchungen zur Selen-Versorgung in der Vorbereitungsfütterung hochträchtiger Milchkühe. Züchtungskunde, v.75, p.88-100, 2003.

GRACE, N.D. et al. Influence of Se status on milk Se concentrations in dairy cows. New Zealand Journal of Agricultural Research, v.40, p.75-78, 1997.

GUO, X.; WU, L. Distribution of free seleno-amino acids in plant tissue of Melilotus indica L. grown in selenium-laden soils. Ecotoxicology and Environmental Safety, v.39, p.207214, 1998

HARTFIEL, W.; SCHULTE, W. Selenmangel in der Bundesrepublik (II). Aktuelle Ernährungsmedizin, v.13, p.77-82, 1988.

HIDIROGLOU, M.; LESSARD, J.R. The effect of selenium or vitamin E supplementation on volatile fatty acid content of rumen liquor in sheep fed a purified diet. International Journal for Vitamin and Nutritional Research, v.46, p.458-463, 1976

JANGHORBANI, M. et al. The selenite-exchangeable metabolic pool in humans: a new concept for the assessment of selenium status. American Journal of Clinical Nutrition, v.51, p.670-677, 1990.

JUKOLA, E. et al. Blood selenium, vitamin E, vitamin A, and ßcarotene concentrations and udder health, fertility treatments, and fertility. Journal of Dairy Science, v.79, p.838-845, 1996.

KABATA-PENDIAS, A. Geochemistry of selenium. Journal of Environmental Pathology, Toxicology and Oncology, v.17, p.173-177, 1998.

KIM, H. et al. Studies on the effects of selenium on rumen microbial fermentation in vitro. Biological Trace Element Research, v.56, p.203-213, 1997

KIRCHGESSNER, M. Underwood memorial lecture Homeostasis and homeorhesis in trace element metabolism. In: ANKE, M. et al. INTERNATIONAL SYMPOSIUM ON TRACE ELEMENTS IN MAN AND ANIMALS, 1993, Jena, Germany. Proceedings... Jena: Verlag Media Touristik, 1993. p.4-12. TEMA-8.

KIRCHGESSNER, M. et al. Homeostatic adjustments of selenium metabolism and tissue selenium to widely varying selenium supply in ${ }^{75}$ Se labeled rats. Journal of Animal 
Physiology and Animal Nutrition, Berlin, v.78, p.20-30, 1997.

KNOWLES, S.O. et al. Significance of amount and form of dietary selenium on blood, milk, and casein selenium concentrations in grazing cows. Journal of Dairy Science, v.82, p.429-437, 1999.

KOENIG, K.M. et al. Effects of diet and chemical form of selenium on selenium metabolism in sheep. Journal of Animal Science, v.75, p.817-827, 1997.

LACETERA, N. et al. Effects of selenium and vitamin E administration during a late stage of pregnancy on colostrum and milk production in dairy cows, and on passive immunity and growth of their offspring. American Journal of Veterinary Research, v.57, p.1776-1780, 1996.

LUCCI, C.S. et al. Selênio em bovinos leiteiros do Estado de São Paulo. I. Níveis de selênio em soros sanguíneos. Revista da Faculdade de Medicina Veterinária e Zootecnia, v.21, n.1, p.65-70, 1984a.

LUCCI, C.S. et al. Selênio em bovinos leiteiros do Estado de São Paulo. II. Níveis de selênio nas forragens e concentrados. Revista da Faculdade de Medicina Veterinária e Zootecnia, v.21, n.1, p.71-76, 1984b.

MALBE, M. et al. Comparisons of selenite and selenium yeast feed supplements on Se-incorporation, mastitis and leucocyte function in Se-deficient dairy cows. Zentralblatt für Veterinärmedizin, Reihe A, v.42, p.111-121, 1995.

MAUS, R.W. et al. Relationship of dietary selenium in plasma and milk from dairy cows. Journal of Dairy Science, v.63, p.532-537, 1980.

MEYER, H. et al. Untersuchungen zur Selenversorgung von Pferden in Norddeutschland. Pferdeheilkunde, v.11, p.313321, 1995.

MILLER, J.K. et al. Oxidative stress, antioxidants, and animal function. Journal of Dairy Science, v.76, p.2812-2823, 1993.

MORAES, S.S. et al. Microelement deficiencies and imbalances in cattle and sheep in some regions of Brazil. Pesquisa Veterinária Brasileira, v.19, n.1, p.19-33, 1999.

MUÑIZ-NAVEIRO, Ó. et al. Selenium content and distribution in cow's milk supplemented with two dietary selenium sources. Journal of Agricultural and Food Chemistry, v.53, p.98179822, 2005.

NRC, National Research Council. Nutrient requirements of dairy cattle. 7.ed. Washington, D.C., 2001. 381p.

NOCKELS, C.F. Antioxidants improve cattle immunity following stress. Animal Feed Science and Technology, v.62, p.59-68, 1996.

$\mathrm{OH}, \mathrm{S} . \mathrm{H}$. et al. Selenium as a component of glutathione peroxidase isolated from ovine erythrocytes. Biochemistry, v.13, p.1825-1829, 1974.

ORTMAN, K.; PEHRSON, B. Selenite and selenium yeast as feed supplements for dairy cows. Zentralblatt für Veterinärmedizin, Reihe A, v.44, p.373-380, 1997.
PASCHOAL, J.J. et al. Suplementação de selênio e vitamina E sobre a contagem de células somáticas no leite de vacas da raça holandesa. Revista Brasileira de Zootecnia, v. 32, n.6, p.2032-2039, 2003. (Supl. 2).

PULS, R. Mineral levels in animal health. 2.ed. Clearbrook: Sherpa International, 1994. p.83-109.

ROVER Jr., L. et al. Sistema antioxidante envolvendo o ciclo metabólico da glutationa associado a métodos eletroanalíticos na avaliação do estress oxidativo. Química Nova, v.24, p.112119, 2001.

SCHÄFER, K.; WOLLGIEN, D. Selengehalte in Einzelfuttermitteln. Landwirtschaftliche Forschung, v.39, p.128-132, 1986.

SCHWARZ, F.J. et al. Cobalt requirement of beef cattle - feed intake and growth at different levels of cobalt supply. Journal of Animal Physiology and Animal Nutrition, v.83, p.121131, 2000.

SHAMBERGER, R.J. Biochemistry of selenium. New York; Plenum, 1983. 334p.

STEVENS, J.B. et al. Serum selenium concentrations and glutathione peroxidase activities in cattle grazing forages of various selenium concentrations. American Journal of Veterinary Research, v.46, p.1556-1561, 1985.

SUNDE, R.A.; HOEKSTRA, W.G. Incorporation of selenium from selenite and selenocystine into glutathione peroxidase in the isolated perfused rat liver. Biochemical and Biophysical Research Communications, v.93, p.1181-1188, 1980.

SUZUKI, K.T. Metabolomics of selenium: Se metabolites based on speciation studies. Journal of Health Science, v.51, p.107-114, 2005.

TERRY, N. et al. Selenium in higher plants. Annual Review of Plant Physiology and Plant Molecular Biology, v.51, p.401-432, 2000.

UDEN, P.C. et al. Selective detection and identification of Se containing compounds-review and recent developments. Journal of Chromatography A, v.1050, p.85-93, 2004.

UNDERWOOD, E.J.; SUTTLE, N.F. The mineral nutrition of livestock. 3.ed. Wallingford, Reino Unido: CABI Publishing, 1999. p.421-476.

VENDELAND, S.C. et al. Uptake of selenite, selenomethionine and selenate by brush border membrane vesicles isolated from rat small intestine. Biometals, v.7, p.305-312, 1994.

WEISS, W.P. et al. Effect of duration of supplementation of selenium and vitamin E on periparturient dairy cows. Journal of Dairy Science, v.73, p.3187-3194, 1990.

YANG, X. et al. Determination of the selenomethionine content in grain and human blood. Wei Sheng Yen Chiku, Beijing, v.26, p.113-116, 1997.

ZACHARA, B.A. Mammalian selenoproteins. Journal of Trace Elements and Electrolytes Health Disease, v.6, p.137-151, 1992.

Ciência Rural, v.37, n.4, jul-ago, 2007. 